\title{
Melhora da independência funcional após internação para reabilitação de pacientes com sequela de Acidente Vascular Cerebral
}

\author{
Improvement of functional independence after \\ hospitalization for rehabilitation of patients \\ with stroke sequelae
}

\section{Mejora de la independencia funcional tras la hospitalización para la rehabilitación de pacientes con secuelas de Accidente Cerebrovascular}

Juliana Araújo de Morais ${ }^{1}$, Isadora Tavares Almeida ${ }^{2}$, Francyelle Sales de Oliveira Perdigão ${ }^{3}$, Francine Aguilera Rodrigues da Silva ${ }^{4}$, Rayne Ramos Fagundes ${ }^{5}$, Cejane Oliveira Martins Prudente ${ }^{6}$

1.Fisioterapeuta. Escola de Ciências Sociais e da Saúde (ECISS), Pontifícia Universidade Católica de Goiás (PUC Goiás), Goiânia-GO, Brasil. Orcid: https://orcid.org/0000-0001-5031-1155

2.Fisioterapeuta. Escola de Ciências Sociais e da Saúde (ECISS), Pontifícia Universidade Católica de Goiás (PUC Goiás), Goiânia-GO, Brasil. Orcid: https://orcid.org/0000-0002-2890-1121

3. Fisioterapeuta. Escola de Ciências Sociais e da Saúde (ECISS), Pontifícia Universidade Católica de Goiás (PUC Goiás), Goiânia-GO, Brasil. Orcid: https://orcid.org/0000-0001-8712-6543

4.Fisioterapeuta, Mestre, Docente do curso de Fisioterapia, Escola de Ciências Sociais e da Saúde (ECISS), Pontifícia Universidade Católica de Goiás (PUC Goiás), Goiânia-GO, Brasil. Orcid: https://orcid.org/00000003-1368-7924

5.Fisioterapeuta, Mestre, Docente do curso de Fisioterapia, Escola de Ciências Sociais e da Saúde (ECISS), Pontifícia Universidade Católica de Goiás (PUC Goiás), Goiânia-GO, Brasil. Orcid: https://orcid.org/00000002-7090-1336

6.Fisioterapeuta, Doutora, Docente do curso de Fisioterapia, Pontifícia Universidade Católica de Goiás (PUC Goiás) e Universidade Estadual de Goiás (UEG), Goiânia-GO, Brasil. Orcid: https://orcid.org/0000-0001$\underline{6499-3011}$

\begin{abstract}
Resumo
Introdução. O Acidente Vascular Cerebral (AVC) é uma das principais causas de incapacidade; e a reabilitação intensiva tem papel importante na melhora da funcionalidade. Objetivo. Comparar a independência funcional de pacientes com sequela de AVC antes e após a internação para reabilitação, relacionando com características sociodemográficas e clínicas. Método. Estudo Iongitudinal, retrospectivo. Foram coletados dados sociodemográficos, clínicos e a Medida da Independência Funcional (MIF) na admissão e alta, de prontuários de 167 pacientes com diagnóstico de AVC internados para reabilitação em um centro de referência, entre julho de 2016 a dezembro de 2018. Resultados. A maior parte era do sexo masculino, com idade maior ou igual a 60 anos. Os pacientes tiveram melhora no escore total, domínios motor e cognitivo da MIF após internação para reabilitação $(p<0,001)$. Houve diminuição do número de pacientes com dependência modificada e aumento com independência completa, principalmente em indivíduos do sexo masculino, com idade inferior a 60 anos, tempo de internação maior que 30 dias e tempo de AVC menor ou igual a 180 dias $(p<0,001)$. Conclusão. A internação para reabilitação melhora a independência funcional de pacientes com sequela de AVC.
\end{abstract}

Unitermos. Acidente Vascular Cerebral, Reabilitação Hospitalar, Avaliação da Deficiência 


\begin{abstract}
Introduction. Stroke is one of the main causes of disability; and intensive rehabilitation plays an important role in improving functionality. Objective. To compare the functional independence of patients with stroke sequelae before and after hospitalization for rehabilitation, relating to sociodemographic and clinical characteristics. Method. Longitudinal and retrospective study. Sociodemographic and clinical data and the Functional Independence Measure (FIM) were collected on admission and discharge from medical records of 167 patients diagnosed with stroke admitted for rehabilitation at the reference center, between July 2016 and December 2018 Result. Most of them were male, aged over 60 years. Patients had improvement in the total FIM score, motor, and cognitive domains after hospitalization for rehabilitation $(p<0.001)$. There was a decrease in the number of patients with modified dependence and an increase with complete independence, especially in males, aged under 60 years, hospital stay longer than 30 days and stroke duration less than or equal to 180 days $(p<0.001)$. Conclusion. Hospitalization for rehabilitation improves the functional independence of patients with stroke sequelae.
\end{abstract}

Keywords. Stroke, Rehabilitation Hospitals, Disability Evaluation

\begin{abstract}
Resumen
Introducción. El Accidente Vascular Cerebral (ACV) es una de las principales causas de discapacidad; y la rehabilitación intensiva juega un papel importante en la mejora de la funcionalidad. Objetivo. Comparar la independencia funcional de los pacientes con secuelas de ictus antes y después de la hospitalización para rehabilitación, en relación con las características sociodemográficas y clínicas. Método. estudio longitudinal, retrospectivo. Se recogieron datos sociodemográficos, clínicos y de independencia funcional (FIM) al ingreso y egreso de las historias clínicas de 167 pacientes con diagnóstico de ictus ingresados para rehabilitación en un centro de referencia, entre julio de 2016 y diciembre de 2018. Resultados. La mayoría eran hombres mayores de 60 años. Los pacientes tuvieron una mejoría en la puntuación total de la FIM, los dominios motor y cognitivo después del ingreso para rehabilitación $(p<0,001)$. Hubo una disminución en el número de pacientes con dependencia modificada y un aumento con total independencia, especialmente en varones, menores de 60 años, estancia hospitalaria mayor de 30 días y duración del ictus menor o igual a 180 días $(p<0,001)$. Conclusión. La hospitalización para rehabilitación mejora la independencia funcional de los pacientes con secuelas de ictus.
\end{abstract}

Palabras clave. Ictus, Hospitales de Rehabilitación, Evaluación de la Discapacidad

Trabalho realizado na Escola de Ciências Sociais e da Saúde (ECISS), Pontifícia Universidade Católica de Goiás (PUC Goiás), Goiânia-GO, Brasil.

Conflito de interesse: não

Recebido em: 30/06/2021

Aceito em: 10/11/2021

Endereço para correspondência: Juliana Araújo de Morais. Pontifícia Universidade Católica de Goiás. Av. Universitária 1.440. Setor Universitário. CEP 74605-010. Goiânia-GO, Brasil. E-mail: jumorais129@gmail.com

\title{
INTRODUÇÃO
}

\author{
O Acidente Vascular Cerebral (AVC) é uma das \\ principais causas de morte e incapacidade no mundo. Em \\ 2016, ocorreram 13,7 milhões de novos casos e 5,5 milhões
} de mortes devido ao AVC1. No Brasil, as doenças cerebrovasculares foram uma das principais causas de morte em 2015 , sendo a taxa de mortalidade de 88,0 por 100 mil 
habitantes ${ }^{2}$. Nos dados da Pesquisa Nacional de Saúde de 2013 foi estimado 2.231 milhões de casos de AVC, sendo que 568 mil tinham deficiências graves. As características sociodemográficas de pacientes acometidos pelo AVC têm predomínio na idade avançada, baixa escolaridade e residentes em zona urbana, e no que diz respeito à incapacidade funcional, não observa-se um perfil sociodemográfico específico, mas há porcentagem maior de incapacidade nos homens $(29,5 \%)$ em comparação com as mulheres $(21,5 \%)^{3}$.

Muitos sobreviventes do AVC possuem sequelas cognitiva, motora, sensitiva e de linguagem, gerando declínio da funcionalidade, limitação nas atividades de vida diária e na participação social, afetando a qualidade de vida e a autonomia4,5. Quanto aos problemas motores, pode-se observar alterações no tônus e no controle motor, fraqueza muscular e contratura muscular, que afetam tarefas como preensão, alcance e manuseio de objetos ${ }^{6}$.

As deficiências motoras e cognitivas após o AVC são muitas vezes persistentes e incapacitantes e podem levar à dependência e institucionalização7,8. A reabilitação assume um papel necessário na recuperação dos indivíduos sobreviventes do AVC e visa melhorar a independência funcional9.

A base fisiológica para a recuperação após o AVC é a neuroplasticidade, que envolve 0 reaprendizado das habilidades motoras e cognitivas que foram perdidas após a lesão ${ }^{10}$. A reabilitação intensiva promove ganhos funcionais 
graduais e contínuos, melhora o desempenho funcional e as atividades de vida diária ${ }^{11}$. Estudos anteriores abordaram essa modalidade de internação para reabilitação ${ }^{12-19}$, mas poucos foram conduzidos no Brasil ${ }^{12,14}$.

Além disso, infelizmente essa ainda não é a realidade de muitas cidades, onde os pacientes demoram longos períodos para iniciar a reabilitação após o AVC, iniciando as terapias já com deformidades e complicações secundária, e ainda recebem tratamento apenas uma ou duas vezes por semana, dificultando a melhora de sua independência funcional.

Dessa forma, o objetivo desse estudo foi comparar a independência funcional de pacientes com sequela de AVC antes e após a internação para reabilitação, relacionando com características sociodemográficas e clínicas (idade, sexo, tempo de AVC e de internação).

\section{MÉTODO}

\section{Amostra}

Trata-se de um estudo quantitativo, retrospectivo e longitudinal. A amostra foi composta por 167 pacientes com sequelas de AVC, principalmente nas fases subaguda ou crônica, internados para reabilitação em uma instituição estadual de reabilitação de Goiânia-GO.

Esta instituição é reconhecida pelo Ministério da Saúde como um Centro Especializado em Reabilitação (CER IV), por sua atuação em reabilitar pessoas com deficiência física, auditiva, visual e intelectual. Possui acreditação de ONA nível 
3 e o Sistema de Gestão da Qualidade. Atende exclusivamente pelo Sistema Único de Saúde e oferece atendimento de alta complexidade.

O regime de hospitalização nesta instituição engloba todos os atendimentos e procedimentos para obter ou complementar o diagnóstico e as terapêuticas necessárias no âmbito hospitalar. A equipe mantém escalas de plantão cobrindo as 24 horas do dia, com cuidados aos pacientes internados para tratamento cirúrgico, clínico ou de reabilitação

$\mathrm{Na}$ modalidade internação para reabilitação, os pacientes são hospitalizados para reabilitação intensiva, estando principalmente nas fases subaguda ou crônica. O atendimento é especializado, composto por uma equipe multiprofissional, que engloba médicos de várias especialidades, enfermeiros, fisioterapeutas, terapeutas ocupacionais, psicólogos, fonoaudiólogos, nutricionistas e assistentes sociais.

Os atendimentos ocorrem diariamente, nos períodos matutino e vespertino, com exceção dos finais de semana, que aos sábados ocorrem no período matutino e aos domingos orientações. Além destes atendimentos, os pacientes participam juntamente com seus cuidadores de um curso de capacitação, com aulas teóricas e práticas. O tempo de internação segue a necessidade de cada paciente; nesta amostra o tempo médio foi de $32,75+10,49$ dias.

Em relação à fisioterapia e terapia ocupacional, os pacientes realizam duas sessões diárias de 30 minutos de 
cada terapia, cinco vezes por semana e aos finais de semana uma sessão. Quanto ao curso de capacitação, cada aula tem duração de aproximadamente uma hora, realizada semanalmente, por aproximadamente cinco semanas.

Foram incluídos no estudo prontuários de pacientes com diagnóstico clínico de AVC, internados para reabilitação entre julho de 2016 a dezembro de 2018, com a Medida de Independência Funcional (MIF) preenchida corretamente no primeiro e último dia de internação hospitalar; e foram excluídos os prontuários dos pacientes com outras doenças neurológicas ou doença incapacitante prévia.

Foi solicitada e aceita a dispensa do Termo de Consentimento Livre e Esclarecido (TCLE) para os Comitês de Ética, por serem dados secundários (prontuários) e não ser possível contactar os pacientes, visto que já receberam alta. O estudo seguiu as normas da Resolução 466/12 do Conselho Nacional de Saúde e foi aprovado pelo Comitê de Ética em Pesquisa da Pontifícia Universidade Católica de Goiás (PUC Goiás) e Comitê de Ética do Centro de Excelência em ensino, pesquisa e projetos Leide das Neves Ferreira, com os pareceres de números 2.966.161, 3.977.915 e 4.038.194.

\section{Procedimento}

Foram utilizados os seguintes instrumentos: Ficha de perfil sociodemográfico e clínico de pacientes com sequela de AVC e a Escala de Medida de Independência Funcional (MIF). 
A ficha de perfil sociodemográfico e clínico foi elaborado pelas pesquisadoras.

A MIF é um instrumento de avaliação quantitativa, sendo seu principal objetivo avaliar a capacidade funcional, o autocuidado e as atividades motoras e cognitivas das atividades de vida diária (AVD's). O instrumento é dividido em dois domínios, motor e cognitivo, avaliados de acordo com as atividades, sendo elas, autocuidado, transferências, locomoção, controle esfincteriano, comunicação e cognição social, incluindo memória, interação social e resolução de problemas. Cada atividade recebe uma pontuação de 1 (dependência total) a 7 (independência completa), portanto o escore total varia de 18 a $126^{20}$. Cada domínio é somado e os níveis de dependência são classificados de acordo com escore total da MIF em 18 pontos: dependência completa; 19 a 60: dependência modificada (assistência de até 50\% das tarefas); 61 a 103: dependência modificada (assistência de até $25 \%$ das tarefas); e 104 a 126: independência completa/modificada ${ }^{21}$. No presente estudo, optou-se por classificar em três categorias: dependência completa, dependência modificada e independência completa.

Os prontuários foram selecionados e analisados de forma eletrônica e os dados foram preenchidos manualmente para os instrumentos do estudo. A partir de uma lista com 741 pacientes com diagnóstico de doenças neurológicas internados para reabilitação na instituição, no período de julho de 2016 a dezembro de 2018, 246 tinham diagnóstico de AVC e destes 79 foram excluídos (16 por outras doenças 
incapacitantes associadas, 36 por terem AVC prévio, dois por dados incompletos, 23 por preenchimento incorreto da MIF e dois por complicações na internação, sendo transferidos para UTI). Assim, a amostra final foi de 167 pacientes.

\section{Análise Estatística}

Os dados foram analisados utilizando o Statistical Package for Social Science (SPSS), versão 26. A descrição do perfil sociodemográfico e clínico foi realizada por meio de frequência absoluta $(n)$ e relativa (\%) e estatísticas descritivas. A normalidade dos dados foi verificada utilizando o teste de Shapiro-Wilk. A comparação da MIF inicial e final foi feita utilizando o teste de Wilcoxon e Qui-quadrado Post hoc. Para todas as análises foi adotado um nível de significância de 5\% $(p<0,05)$.

\section{RESULTADOS}

Participaram do estudo 167 pacientes com sequela de AVC, com idade mínima de 13, máxima de 96 e média de 57,76 $\pm 15,84$ anos. A Tabela 1 apresenta 0 perfil sociodemográfico dos participantes, sendo a maior parte do sexo masculino, com idade maior ou igual a 60 anos, casado, com ensino fundamental e renda mensal de até três saláriosmínimos.

A Tabela 2 descreve os dados clínicos da amostra. O tempo médio de internação dos pacientes foi de $32,75 \pm 10,49$ dias. Já o tempo médio de AVC foi de 
$146,11 \pm 163,63$, houve maior frequência de tempo de internação superior a 30 dias e tempo de AVC inferior a 90 dias. A maior parte dos pacientes tinha AVC isquêmico, hemiplegia, espástica, à esquerda. Uma minoria foi submetida a procedimento cirúrgico. Quanto às patologias associadas relatadas nos prontuários dos pacientes, 63,5\% tinham hipertensão arterial sistêmica, 26,3\% diabetes mellitus e $27,5 \%$ doenças cardíacas.

Tabela 1. Caracterização do perfil sociodemográfico de pacientes com sequela de Acidente Vascular Cerebral.

\begin{tabular}{lcc}
\hline & $\mathbf{n}$ & $\mathbf{\%}$ \\
\hline Sexo & 71 & 42,5 \\
$\quad$ Feminino & 96 & 57,5 \\
$\quad$ Masculino & & \\
Faixa etária (anos) & 76 & 45,5 \\
$\quad<60$ & 91 & 54,5 \\
$\quad \geq 60$ & & \\
Estado Civil & 76 & 45,5 \\
$\quad$ Casado & 19 & 11,4 \\
$\quad$ Divorciado & 39 & 23,4 \\
Solteiro & 13 & 7,8 \\
$\quad$ União estável & 19 & 11,4 \\
$\quad$ Viúvo & 1 & 0,6 \\
$\quad$ Não informado & & \\
Escolaridade & 20 & 12,0 \\
$\quad$ Não alfabetizado & 71 & 42,5 \\
$\quad$ Ensino fundamental & 30 & 18,0 \\
Ensino médio & 31 & 18,6 \\
$\quad$ Ensino superior & 15 & 9,0 \\
$\quad$ Não informado & & \\
Renda mensal (salários-mínimos) & 61 & 36,5 \\
$\quad 1$ & 55 & 32,9 \\
2 a 3 & 32 & 19,2 \\
$\quad \geq 4$ & 19 & 11,4 \\
$\quad$ Não informado & & \\
\hline $\mathrm{n}$ = frequência absoluta; \% frequência relativa & &
\end{tabular}


Tabela 2. Descrição dos dados clínicos e patologias associadas dos pacientes com sequela de Acidente Vascular Cerebral.

\begin{tabular}{|c|c|c|}
\hline & $\mathbf{n}$ & $\%$ \\
\hline \multicolumn{3}{|l|}{ Tempo de internação } \\
\hline$\leq 30$ dias & 63 & 37,7 \\
\hline$>30$ dias & 104 & 62,3 \\
\hline \multicolumn{3}{|l|}{ Tempo da lesão } \\
\hline$<90$ dias & 82 & 49,1 \\
\hline 90 a 180 dias & 47 & 28,1 \\
\hline$>180$ dias & 38 & 22,8 \\
\hline \multicolumn{3}{|c|}{ Tipo de Acidente Vascular Cerebral } \\
\hline Hemorrágico & 45 & 26,9 \\
\hline Hemorrágico/Isquêmico & 3 & 1,8 \\
\hline Isquêmico & 118 & 70,7 \\
\hline Não informado & 1 & 0,6 \\
\hline \multicolumn{3}{|l|}{ Diagnóstico funcional } \\
\hline Hemiplegia & 150 & 89,8 \\
\hline Tetraplegia & 17 & 10,2 \\
\hline \multicolumn{3}{|l|}{ Tônus } \\
\hline Espástico & 86 & 51,5 \\
\hline Flácido & 4 & 2,4 \\
\hline Normal & 54 & 32,3 \\
\hline Não informado & 23 & 13,8 \\
\hline \multicolumn{3}{|l|}{ Lado acometido } \\
\hline Direito & 70 & 47,0 \\
\hline Esquerdo & 80 & 53,0 \\
\hline \multicolumn{3}{|l|}{ Procedimento cirúrgico } \\
\hline Não & 119 & 71,3 \\
\hline $\operatorname{Sim}$ & 48 & 28,7 \\
\hline \multicolumn{3}{|c|}{ Hipertensão Arterial Sistêmica } \\
\hline Não & 16 & 9,6 \\
\hline Sim & 106 & 63,5 \\
\hline Não informado & 45 & 26,9 \\
\hline \multicolumn{3}{|l|}{ Diabetes Mellitus } \\
\hline Não & 42 & 25,1 \\
\hline Sim & 44 & 26,3 \\
\hline Não informado & 81 & 48,5 \\
\hline \multicolumn{3}{|l|}{ Doença cardíaca } \\
\hline Não & 30 & 18,0 \\
\hline $\operatorname{Sim}$ & 46 & 27,5 \\
\hline Não informado & 91 & 54,5 \\
\hline
\end{tabular}


sensibilidade. Da amostra total, apenas $24,6 \%$ dos participantes deambulavam e $88 \%$ utilizavam cadeira de rodas.

A Tabela 3 compara os domínios, pontuação total e classificação da MIF antes e após internação para reabilitação. Observa-se que houve diferença significante nos domínios e pontuação total; diminuição no número de pacientes com dependência modificada e aumento dos com independência completa.

Tabela 3. Comparação dos domínios, escore total e classificação da MIF inicial e final.

\begin{tabular}{|c|c|c|c|c|}
\hline \multirow[t]{2}{*}{ MIF } & \multicolumn{2}{|c|}{ Média \pm DP } & \multirow{2}{*}{$z$} & \multirow{2}{*}{ p* } \\
\hline & Inicial & Final & & \\
\hline \multicolumn{5}{|l|}{ Domínios } \\
\hline Motor & $33,87 \pm 16,15$ & $50,17 \pm 21,30$ & 10,35 & $<0,001$ \\
\hline Cognitivo & $19,95 \pm 8,18$ & $23,14 \pm 8,27$ & 8,32 & $<0,001$ \\
\hline \multirow[t]{3}{*}{ Escore total } & $53,78 \pm 27,05$ & $72,99 \pm 28,14$ & 10,40 & $<0,001$ \\
\hline & \multicolumn{2}{|c|}{ n (\%) } & $z$ & $p * *$ \\
\hline & Inicial & Final & & \\
\hline \multicolumn{5}{|l|}{ Classificação } \\
\hline Dependência completa & $3(1,8)$ & $2(1,2)$ & 0,20 & 0,44 \\
\hline Dependência modificada & $161(96,4)$ & $139(83,2)$ & 15,84 & 0,01 \\
\hline Independência completa & $3(1,8)$ & $26(15,6)$ & 19,97 & $<0,001$ \\
\hline
\end{tabular}

A Tabela 4 apresenta o resultado da comparação da classificação da MIF em relação ao sexo, faixa etária, tempo de internação e tempo de AVC. Observa-se que houve melhora em pelo menos uma das classificações da MIF em todos os grupos analisados; mas chama a atenção, com $\mathrm{p}<0,001$, na diminuição do número de pacientes com 
dependência modificada e aumento dos com independência completa em indivíduos do sexo masculino, com idade inferior a 60 anos, tempo de internação maior que 30 dias e tempo de AVC menor ou igual a 180 dias.

Tabela 4. Comparação da classificação da MIF inicial e final segundo o sexo, faixa etária, tempo de internação e de AVC.

\begin{tabular}{|c|c|c|c|c|}
\hline & \multicolumn{2}{|c|}{ Intervenção n (\%) } & \multirow{2}{*}{$x^{2}$} & \multirow[b]{2}{*}{$p^{*}$} \\
\hline & Inicial & Final & & \\
\hline \multicolumn{5}{|l|}{ Sexo } \\
\hline \multicolumn{5}{|l|}{ Feminino } \\
\hline Dependência completa & $3(4,2)$ & $1(1,4)$ & 1,03 & 0,31 \\
\hline Dependência modificada & $67(94,4)$ & $61(85,9)$ & 2,85 & 0,09 \\
\hline Independência completa & $1(1,4)$ & $9(12,7)$ & 6,88 & 0,01 \\
\hline \multicolumn{5}{|l|}{ Masculino } \\
\hline Dependência completa & $0(0,0)$ & $1(1,0)$ & 1,01 & 0,32 \\
\hline Dependência modificada & $94(97,9)$ & $78(81,3)$ & 14,29 & $<0,001$ \\
\hline Independência completa & $2(2,1)$ & $17(17,7)$ & 13,14 & $<0,001$ \\
\hline \multicolumn{5}{|l|}{ Faixa etária } \\
\hline \multicolumn{5}{|l|}{$<60$} \\
\hline Dependência completa & $1(1,3)$ & $0(0,0)$ & 1,01 & 0,32 \\
\hline Dependência modificada & $72(94,7)$ & $59(77,6)$ & 9,34 & $<0,001$ \\
\hline Independência completa & $3(3,9)$ & $17(22,4)$ & 11,28 & $<0,001$ \\
\hline \multicolumn{5}{|l|}{$\geq 60$} \\
\hline Dependência completa & $2(2,2)$ & $2(2,2)$ & 0,00 & 1,00 \\
\hline Dependência modificada & $89(97,8)$ & $80(87,9)$ & 6,71 & 0,01 \\
\hline Independência completa & $0(0,0)$ & $9(9,9)$ & 9,47 & $<0,001$ \\
\hline \multicolumn{5}{|l|}{ Tempo de internação } \\
\hline \multicolumn{5}{|l|}{$\leq 30$ dias } \\
\hline Dependência completa & $2(3,2)$ & $0(0,0)$ & 2,03 & 0,15 \\
\hline Dependência modificada & $59(93,7)$ & $52(82,5)$ & 3,71 & 0,05 \\
\hline Independência completa & $2(3,2)$ & $11(17,5)$ & 6,95 & 0,01 \\
\hline \multicolumn{5}{|l|}{$>30$ dias } \\
\hline Dependência completa & $1(1,0)$ & $2(1,9)$ & ,34 & 0,56 \\
\hline Dependência modificada & $102(98,1)$ & $87(83,7)$ & 13,03 & $<0,001$ \\
\hline Independência completa & $1(1,0)$ & $15(14,4)$ & 13,27 & $<0,001$ \\
\hline \multicolumn{5}{|l|}{ Tempo da AVC } \\
\hline \multicolumn{5}{|l|}{$\leq 180$ dias } \\
\hline Dependência completa & $3(2,3)$ & $1(0,8)$ & 1,02 & 0,31 \\
\hline Dependência modificada & $123(95,3)$ & $106(82,2)$ & 11,23 & $<0,001$ \\
\hline Independência completa & $3(2,3)$ & $22(17,1)$ & 15,99 & $<0,001$ \\
\hline \multicolumn{5}{|l|}{$>180$ dias } \\
\hline Dependência completa & $0(0,0)$ & $1(2,6)$ & 1,01 & 0,31 \\
\hline Dependência modificada & $38(100,0)$ & $33(86,8)$ & 5,35 & 0,02 \\
\hline Independência completa & $0(0,0)$ & $4(10,5)$ & 4,22 & 0,04 \\
\hline
\end{tabular}

*Qui-quadrado Posthoc; $\mathrm{n}$ = frequência absoluta; \% = frequência relativa 


\section{DISCUSSÃO}

Os resultados desse estudo evidenciaram que houve melhora funcional dos pacientes com sequela de AVC após internação para reabilitação, corroborando com achados de outros estudos ${ }^{12-19}$. A média da MIF total inicial foi de 53,78 e a final 72,99, com diferença de 19,21. Outros estudos, também com pacientes com sequela de AVC internados para reabilitação, obtiveram ganhos funcionais na MIF total pouco superior ao presente estudo ${ }^{13,16}$.

A média da MIF motora inicial foi de 33,87 e a final de 50,17 . Um estudo que analisou o efeito do programa de internação para reabilitação obteve escore médio aproximado da MIF motora inicial de 35,2 e o final mais baixo que o presente estudo nos pacientes com sequela de AVC, de 44,9, demonstrando menos ganho neste domínio, os pacientes foram submetidos a um programa com cinco dias de terapias por semana, com média de internação de $30,9 \pm 15,2$ dias $^{14}$. Vale destacar que no presente estudo os pacientes fizeram terapias também nos finais de semana e 0 tempo de internação foi de $32,75 \pm 10,49$ dias, o que pode ter influenciado nos resultados.

Quanto a MIF cognitiva, a média inicial foi de 19,95 e a final de 23,14. Um estudo retrospectivo realizado em um hospital ao norte da Califórnia obteve média inicial mais baixa $(16,3)$ e a final aproximada $(22,5)^{16}$. 0 artigo que analisou o banco de dados de reabilitação do Japão, com amostra de 2325 pacientes, também teve valores aproximados, sendo a admissão 20,2 e na alta o grupo com 
mais de 15 horas de reabilitação foi de 24 e com menos de 15 horas de 23,613.

A recuperação após o AVC está diretamente relacionada a neuroplasticidade, através do desenvolvimento de novas conexões neuronais, recuperando assim os déficits sensoriais e motores ${ }^{22}$. O aprendizado de habilidades motoras repetidas e a aprendizagem específica de tarefas têm capacidade de alterar a ativação cortical, estimulando a mudança neuroplástica e induzindo o padrão normal e contralateral da ativação cerebral após o $\mathrm{AVC}^{23}$.

A reabilitação multiprofissional intensiva tem como benefícios maximizar a funcionalidade do paciente com sequela de AVC em um curto espaço de tempo, diminuindo a permanência nas unidades de reabilitação ${ }^{12,17}$. Um estudo que comparou os pacientes internados para reabilitação com os das instalações de enfermagem especializadas, observou que na internação para reabilitação os pacientes tinham menor permanência hospitalar e apresentaram escores da MIF maiores, melhora da mobilidade e autocuidado ${ }^{18}$.

Neste estudo, os pacientes do sexo masculino tiveram diminuição da dependência modificada e aumento na independência completa $(p<0,001)$; já o sexo feminino, houve aumento apenas no número de pacientes com independência completa $(p=0,01)$. Outro estudo identificou que pacientes do sexo feminino têm mais dificuldade na recuperação das sequelas após o AVC agudo do que o sexo masculino ${ }^{24}$. 
As mulheres ficam mais dependentes que os homens após sobreviverem ao AVC ${ }^{25,26}$. A incapacidade é maior nas mulheres, pois são acometidas mais velhas, apresentam mais comorbidades e têm maior gravidade do $\mathrm{AVC}^{25}$.

No presente estudo, tanto o grupo com menos de 60 anos, quanto acima desta idade tiveram diminuição na quantidade de pacientes com dependência modificada e aumento na independência completa após reabilitação, mas houve diminuição do número de pacientes com dependência modificada na faixa etária inferior a 60 anos $(p<0,001)$. Um estudo comparou a recuperação funcional de idosos internados para reabilitação e identificou que quanto maior a idade menor a evolução funcional dos pacientes com sequela de $\mathrm{AVC}^{19}$.

Com o envelhecimento existem perdas funcionais advindas da própria idade. Mesmo com o declínio da capacidade funcional dos idosos há necessidade de investir em estratégias de reabilitação que venham amenizar as perdas funcionais e, principalmente, das sequelas do $A V C^{27}$. A função cognitiva diminui com o aumento da idade ${ }^{19}$. Porém, apesar do comprometimento cognitivo os pacientes obtêm ganhos funcionais significativos durante a reabilitação 28 .

Pacientes internados por mais de 30 dias tiveram diminuição da dependência modificada e aumento da independência completa $(p<0,001)$. Já o grupo que permaneceu menos que 30 dias, apresentou apenas aumento da independência completa $(p=0,01)$. Um estudo 
que comparou as instalações de internação para reabilitação (terapia intensiva) com as instalações de reabilitação usual, observou que o primeiro grupo apresentou tempo de permanência hospitalar maior (109,2 239,7 dias), receberam mais terapia e obtiveram mais ganhos funcionais ${ }^{13}$. Vale destacar que o tempo médio de internação dos pacientes do presente estudo foi de $32,75 \pm 10,49$ dias.

Uma pesquisa identificou que o tratamento de alta intensidade e longa duração possibilitou ganhos mais eficazes no desempenho de tarefas funcionais, indicando benefício com mais de 150 horas de terapia ${ }^{29}$. É importante considerar que o tempo de reabilitação pode ser afetado pelas características do paciente, como o lado acometido, gravidade das condições médicas e estado funcional inicial ${ }^{13}$.

Pacientes com tempo inferior ou igual a 180 dias de lesão tiveram melhora, após internação, para reabilitação na diminuição da dependência modificada e aumento da independência completa $(p<0,001)$. Outros estudos também identificaram que quanto mais precoce a admissão na internação para reabilitação, melhores os resultados funcionais ${ }^{15,16}$. A recuperação funcional substancial pode começar após as primeiras semanas do AVC, apesar disto mais de $50 \%$ dos sobreviventes de AVC possuem hemiparesia após seis meses ${ }^{30}$.

\section{CONCLUSÃO}

A internação para reabilitação traz efeitos positivos na funcionalidade dos pacientes com sequela de AVC. Após esta 
modalidade de tratamento, os pacientes tiveram melhora na pontuação total e nos domínios motor e cognitivo da MIF. Indivíduos do sexo masculino, com idade inferior a 60 anos, tempo de internação maior que 30 dias e tempo de AVC menor ou igual a 180 dias apresentaram diminuição da dependência modificada e aumento da independência completa.

Uma limitação do estudo refere-se ao fato de ser retrospectivo, com base em análise de prontuário. Mesmo diante a melhora funcional importante dos participantes deste estudo, os achados demonstram a necessidade de um olhar diferenciado, com estratégias visando melhor recuperação dos pacientes do sexo feminino, com idade superior a 60 anos e tempo de AVC maior que seis meses.

\section{REFERÊNCIAS}

1.GBD 2016 Stroke Collaborators. Global, regional, and national burden of stroke, 1990-2016: a systematic analysis for the Global Burden of Disease Study 2016. Lancet Neurol 2019;18:439-58. https://doi.org/10.1016/S1474-4422(19)30034-1

2.Brant LCC, Nascimento BR, Passos VMA, Duncan BB, Bensenõr IJM, Malta DC, et al. Variações e diferenciais da mortalidade por doença cardiovascular no Brasil e em seus estados, em 1990 e 2015: estimativas do Estudo Carga Global de Doença. Rev Bras Epidemiol 2017;20(Suppl 1):116-28. https://doi.org/10.1590/19805497201700050010

3.Bensenor IM, Goulart AC, Szwarcwald CL, Vieira MLFP, Malta DC, Lotufo PA. Prevalence of stroke and associated disability in Brazil: National Health Survey - 2013. Arq Neuropsiquiatr 2015;73:746-50. https://doi.org/10.1590/0004-282X20150115

4.Faria-Fortini I, Basílio ML, Scianni AA, Faria CDCM, Teixeira-Salmela LF. Performance and capacity-based measures of locomotion, compared to impairment-based measures, best predicted participation in individuals with hemiparesis due to stroke. Disabil Rehabil 2018;40:1791-8. https://doi.org/10.1080/09638288.2017.1312570 
5. Motta E, Natalio MA, Waltrick PT. Intervenção fisioterapêutica e tempo de internação em pacientes com Acidente Vascular Encefálico. Rev Neurocienc 2008; 16:118-23.

https://doi.org/10.34024/rnc.2008.v16.8648

6. Bleyenheuft $Y$, Gordon AM. Precision grip in congenital and acquired hemiparesis: similarities in impairments and implications for neurorehabilitation. Front Hum Neurosci 2014;8:459. https://doi.org/10.3389/fnhum.2014.00459

7. Barbay M, Taillia H, Nedelec-Ciceri C, Arnoux A, Puy L, Wiener E, et al. Vascular cognitive impairment: Advances and trends. Rev Neurol (Paris) 2017;173:473-80.

https://doi.org/10.1016/j.neurol.2017.06.009.

8. Klamroth-Marganska V. Stroke Rehabilitation: Therapy Robots and Assistive Devices. Adv Exp Med Biol 2018;1065:579-87. https://doi.org/10.1007/978-3-319-77932-4 35

9.Tanlaka E, King-Shier K, Green T, Seneviratne C, Dukelow S. Inpatient Rehabilitation Care in Alberta: How Much Does Stroke Severity and Timing Matter? Can J Neurol Sci 2019;46:691-701. https://doi.org/10.1017/cjn.2019.276

10.Mang CS, Campbell KL, Ross CJ, Boyd LA. Promoting neuroplasticity for motor rehabilitation after stroke: considering the effects of aerobic exercise and genetic variation on brain-derived neurotrophic factor. Phys Ther 2013;93:1707-16. https://doi.org/10.2522/ptj.20130053 11.Daly JJ, McCabe JP, Holcomb J, Monkiewicz M, Gansen J, Pundik S. Long-Dose Intensive Therapy Is Necessary for Strong, Clinically Significant, Upper Limb Functional Gains and Retained Gains in Severe/Moderate Chronic Stroke. Neurorehabil Neural Repair 2019;33:523-37. https://doi.org/10.1177/1545968319846120

12.Filippo TRM, Alfieri FM, Daniel CR, Souza DR, Battistella LR. Modelo de reabilitação hospitalar após acidente vascular cerebral em país em desenvolvimento. Acta Fisiátr 2017;24:44-7. https://doi.org/10.5935/0104-7795.20170009

13.Kamo T, Momosaki R, Suzuki K, Asahi R, Azami M, Ogihara $H$, et al. Effectiveness of Intensive Rehabilitation Therapy on Functional Outcomes After Stroke: A Propensity Score Analysis Based on Japan Rehabilitation Database. J Stroke Cerebrovasc Dis 2019;28:2537-42. https://doi.org/10.1016/j.jstrokecerebrovasdis.2019.06.007

14.Jorge LL, Brito AMDND, Marchi FHG, Hara ACP, Battistella LR, Riberto M. New rehabilitation models for neurologic inpatients in Brazil. Disabil Rehabil 2015;37:268-73.

https://doi.org/10.3109/09638288.2014.914585

15. Horn SD, DeJong G, Smout RJ, Gassaway J, James R, Conroy B. Stroke rehabilitation patients, practice, and outcomes: is earlier and more aggressive therapy better? Arch Phys Med Rehabil 2005;86(Suppl 2):S101-14.

https://doi.org/10.1016/j.apmr.2005.09.016

16. Wang H, Camicia M, Terdiman J, Mannava MK, Sidney S, Sandel ME. Daily treatment time and functional gains of stroke patients during 
inpatient rehabilitation. PM $\mathrm{R}$ 2013;5:122-8. https://doi.org/10.1016/j.pmrj.2012.08.013

17. Bindawas SM, Vennu V, Moftah E. Improved functions and reduced length of stay after inpatient rehabilitation programs in older adults with stroke: A systematic review and meta-analysis of randomized controlled trials. NeuroRehabilitation 2017;40:369-90. https://doi.org/10.3233/NRE-161425

18. Hong I, Goodwin JS, Reistetter TA, Kuo YF, Mallinson T, Karmarkar $A$, et al. Comparison of Functional Status Improvements Among Patients With Stroke Receiving Postacute Care in Inpatient Rehabilitation vs Skilled Nursing Facilities. JAMA 2019;2:e1916646. https://doi.org/10.1001/jamanetworkopen.2019.16646

19.Mutai H, Furukawa T, Wakabayashi A, Suzuki A, Hanihara T. Functional outcomes of inpatient rehabilitation in very elderly patients with stroke: differences across three age groups. Top Stroke Rehabil 2018;25:269-75. https://doi.org/10.1080/10749357.2018.1437936 20.Riberto M, Miyazaki MH, Jucá SSH, Sakamoto H, Pinto PPN, Battistella L. Validação da versão brasileira da Medida de Independência Funcional. Acta Fisiátr 2004;11:72-6. https://doi.org/10.5935/0104-7795.20040003

21.Ricci NA, Kubota MT, Cordeiro RC. Concordância de observações sobre a capacidade funcional de idosos em assistência domiciliar. Rev Saúde Púb 2005;39:655-62. https://doi.org/10.1590/S003489102005000400021

22. Muzaffar T, Kaleem M. Rehabilitation of post stroke patients with a focus on neuroplasticity. Neurorehabil Neural Repair 2018;32(45):415. https://doi.org/10.1177/1545968318765498

23.Boyd LA, Vidoni ED, Wessel BD. Motor learning after stroke: is skill acquisition a prerequisite for contralesional neuroplastic change? Neurosci Lett 2010;482:21-5.

https://doi.org/10.1016/j.neulet.2010.06.082

24.Kim JS, Lee KB, Roh H, Ahn MY, Hwang HW. Gender differences in the functional recovery after acute stroke. J Clin Neurol 2010;6:183-8. https://doi.org/10.3988/jcn.2010.6.4.183

25.Gall SL, Donnan G, Dewey HM, Macdonell R, Sturm J, Gilligan A, et al. Sex differences in presentation, severity, and management of stroke in a population-based study. Neurology 2010;74:975-81. https://doi.org/10.1212/WNL.0b013e3181d5a48f

26.Phan HT, Blizzard CL, Reeves MJ, Thrift AG, Cadilhac DA, Sturm J, et al. Sex Differences in Long-Term Quality of Life Among Survivors After Stroke in the INSTRUCT. Stroke 2019;50:2299-306. https://doi.org/10.1161/STROKEAHA.118.024437

27. Cruz KCTD, Diogo MJDE. Avaliação da capacidade funcional de idosos com acidente vascular encefálico. Acta Paul Enferm 2009;22:666-72.

https://doi.org/10.1590/S0103-

21002009000500011

28. Rabadi MH, Rabadi FM, Edelstein L, Peterson M. Cognitively impaired stroke patients do benefit from admission to an acute 
rehabilitation unit. Arch Phys Med Rehabil 2008;89:441-8. https://doi.org/10.1016/j.apmr.2007.11.014

29.Daly JJ, McCabe JP, Holcomb J, Monkiewicz M, Gansen J, Pundik S. Long-Dose Intensive Therapy Is Necessary for Strong, Clinically Significant, Upper Limb Functional Gains and Retained Gains in Severe/Moderate Chronic Stroke. Neurorehabil Neural Repair 2019;33:523-37. https://doi.org/10.1177/1545968319846120.

30.Bundy DT, Nudo RJ. Preclinical Studies of Neuroplasticity Following Experimental Brain Injury. Stroke 2019;50:2626-33. https://doi.org/10.1161/STROKEAHA.119.023550 